PROCEEDINGS OF THE

AMERICAN MATHEMATICAL SOCIETY

Volume 135, Number 5, May 2007, Pages 1523-1533

S 0002-9939(06)08598-4

Article electronically published on November 13, 2006

\title{
FORCING A MUTUAL STATIONARITY PROPERTY IN COFINALITY $\omega_{1}$
}

\author{
PETER KOEPKE
}

(Communicated by Julia Knight)

\begin{abstract}
We show that the consistency strength, relative to the system $\mathrm{ZFC}$, of the mutual stationarity property $\operatorname{MS}\left(\aleph_{3}, \aleph_{5}, \aleph_{7}, \ldots ; \omega_{1}\right)$ is equal to the existence of one measurable cardinal. We also discuss mutual stationarity for some other configurations of small cardinal parameters.
\end{abstract}

\section{INTRODUCTION}

The concept of mutual stationarity, introduced by M. Foreman and M. Magidor [1, allows us to transfer some combinatorial aspects of stationary sets to singular cardinals.

Definition 1.1. Let $\left(\kappa_{n}\right)_{n<\omega}$ be a strictly increasing sequence of regular cardinals $\geqslant \aleph_{2}$ with $\kappa_{\omega}=\sup _{n<\omega} \kappa_{n}$. A sequence $\left(S_{n}\right)_{n<\omega}$ is called mutually stationary in $\left(\kappa_{n}\right)_{n<\omega}$ if every first-order structure $\mathfrak{A}$ of countable type with $\kappa_{\omega} \subseteq \mathfrak{A}$ has an elementary substructure $B \prec \mathfrak{A}$ such that $\forall n<\omega$ sup $B \cap \kappa_{n} \in S_{n}$.

If $\left(S_{n}\right)_{n<\omega}$ is mutually stationary in $\left(\kappa_{n}\right)_{n<\omega}$, then, by a standard model-theoretic characterisation of stationarity, each $S_{n} \cap \kappa_{n}$ is stationary in $\kappa_{n}$. For the study of the converse one formulates a mutual stationarity property MS. This property is dependent on the cofinalities of the ordinals in the stationary sets $S_{n}$; we denote the class $\{\xi \in \operatorname{Ord} \mid \operatorname{cof}(\xi)=\lambda\}$ by $\operatorname{Cof}_{\lambda}$.

Definition 1.2. Let $\left(\kappa_{n}\right)_{n<\omega}$ be a strictly increasing sequence of regular cardinals and let $\left(\lambda_{n}\right)_{n<\omega}$ be a sequence of regular cardinals such that $\forall n<\omega \lambda_{n}<\kappa_{n}$. The mutual stationarity property $\operatorname{MS}\left(\left(\kappa_{n}\right)_{n<\omega} ;\left(\lambda_{n}\right)_{n<\omega}\right)$ is the statement: if $\left(S_{n}\right)_{n<\omega}$ is a sequence of sets $S_{n} \subseteq \operatorname{Cof}_{\lambda_{n}}$ each of which is stationary in $\kappa_{n}$, then $\left(S_{n}\right)_{n<\omega}$ is mutually stationary in $\left(\kappa_{n}\right)_{n<\omega}$. If $\left(\lambda_{n}\right)_{n<\omega}$ is the constant sequence $\lambda_{n} \equiv \lambda$ we also write $\operatorname{MS}\left(\left(\kappa_{n}\right)_{n<\omega} ; \lambda\right)$.

In cofinality $\omega$ there is a remarkable ZFC result by M. Foreman and M. Magidor [1], whereas in higher cofinalities, and for accessible cardinals $\kappa_{n}$, the mutual stationarity property gains large cardinal strength.

Theorem 1.3. For $\left(\kappa_{n}\right)_{n<\omega}$ a strictly increasing sequence of uncountable regular cardinals, $\operatorname{MS}\left(\left(\kappa_{n}\right)_{n<\omega} ; \omega\right)$ holds.

Received by the editors October 13, 2005 and, in revised form, December 6, 2005.

2000 Mathematics Subject Classification. Primary 03E35; Secondary 03E02.

(C)2006 American Mathematical Society

Reverts to public domain 28 years from publication 
Theorem 1.4 (J. Cummings, M. Foreman and M. Magidor [2]; P. Koepke and P. Welch [5]). The theories ZFC $+\exists\left(\kappa_{n}\right)_{n<\omega} \mathrm{MS}\left(\left(\kappa_{n}\right)_{n<\omega} ; \omega_{1}\right)$ and ZFC $+\exists \kappa \kappa$ measurable are equiconsistent.

Theorem 1.5 (Koepke and Welch [5]).

a) The property $\operatorname{MS}\left(\aleph_{2}, \aleph_{3}, \aleph_{4}, \ldots ; \omega_{1}\right)$ implies that there is an inner model with an inaccessible limit of measurable cardinals. b) The property $\operatorname{MS}\left(\aleph_{3}, \aleph_{5}, \aleph_{7}, \ldots ; \omega_{2}\right)$ implies that there is an inner model with an inaccessible limit of measurable cardinals.

The main result of this paper says that there are, nevertheless, some mutual stationarity properties in cofinality $\omega_{1}$ for particular sequences of $\aleph_{n}$ 's which are equiconsistent with one measurable cardinal:

Theorem 1.6. Let $\kappa$ be a measurable cardinal. Then there is a forcing extension of $V$ in which $\operatorname{MS}\left(\aleph_{3}, \aleph_{5}, \aleph_{7}, \ldots ; \omega_{1}\right)$ holds.

In the construction we adapt an argument of Silver's for forcing Chang's conjecture with the help of Martin's axiom $\mathrm{MA}_{\aleph_{1}}$ (see paragraph 19 of [3]). Other mutual stationarities hold in the extension. Previous authors have forced the following from much stronger large cardinals; see e.g. [6], 7], or [2].

Theorem 1.7. In the above forcing extension $\left(\operatorname{Cof}_{\omega}, \operatorname{Cof}_{\omega_{1}}, \operatorname{Cof}_{\omega}, \operatorname{Cof}_{\omega_{1}}, \ldots\right)$ is mutually stationary in $\left(\aleph_{2}, \aleph_{3}, \aleph_{4}, \aleph_{5}, \ldots\right)$.

\section{The FORCING CONSTRUCTION}

In our forcing extension, we shall prove a combinatorial principle which is equivalent to the model-theoretic characterisation of our mutual stationarity property. A standard coding of a structure $\mathfrak{A} \supseteq \aleph_{\omega}$ by a (Skolem) function $F:\left[\aleph_{\omega}\right]^{<\omega} \rightarrow \aleph_{\omega}$ yields:

Proposition 2.1. $\operatorname{MS}\left(\aleph_{3}, \aleph_{5}, \aleph_{7}, \ldots ; \omega_{1}\right)$ is equivalent to the property: for every function $F:\left[\aleph_{\omega}\right]^{<\omega} \rightarrow \aleph_{\omega}$ and every sequence $\left(S_{n}\right)_{n<\omega}, S_{n}$ stationary in $\aleph_{2 n+3}$, $S_{n} \subseteq \operatorname{cof}_{\omega_{1}}$ there exists a set $X \subseteq \aleph_{\omega}$ such that

$$
\forall n<\omega \sup \left(\left(F^{\prime \prime}[X]^{<\omega}\right) \cap \aleph_{2 n+3}\right) \leqslant \sup \left(X \cap \aleph_{2 n+3}\right) \in S_{n} .
$$

The following partition property will be crucial in the subsequent proof:

Definition 2.2. Let $\kappa_{0}<\kappa_{1}<\ldots$ be a sequence of infinite cardinals with supre$\operatorname{mum} \kappa$.

a) For $x \in[\kappa]^{<\omega}$ let type $(x)=\left(\operatorname{card}\left(x \cap \kappa_{n}\right) \mid n<\omega\right) \in{ }^{\omega} \omega$; type $(x)$ is called the type of $x$. We say that $t \in{ }^{\omega} \omega$ is a type if $t$ is of the form type $(x)$ for some $x \in[\kappa]^{<\omega}$.

b) If $t$ is a type, let $[\kappa]^{t}=\left\{x \in[\kappa]^{<\omega} \mid \operatorname{type}(x)=t\right\}$.

c) For $a \subseteq \omega$ define a projection function $\left\lceil a:[\kappa]^{<\omega} \rightarrow[\kappa]^{<\omega}\right.$ by

$$
x\lceil a=\{\xi \in x \mid \operatorname{otp}(\xi \cap x) \in a\} .
$$

d) Let $F:[\kappa]^{<\omega} \rightarrow \kappa$. A sequence $\left(I_{n}\right)_{n<\omega}$ is mutually homogeneous for $F$ if for all $x, y \in\left[\bigcup_{n<\omega} I_{n}\right]^{<\omega}$ with type $(x)=\operatorname{type}(y)$ :

$$
\text { if } x \cap(F(x)+1)=y \cap(F(x)+1) \text {, then } F(x)=F(y) \text {. }
$$


e) The sequence $\left(\kappa_{n}\right)_{n<\omega}$ is mutually Ramsey if for every function $F:[\kappa]^{<\omega} \rightarrow \kappa$ there exists a mutually homogeneous sequence $\left(I_{n}\right)_{n<\omega}$ such that $\operatorname{card}\left(X_{n}\right)=\kappa_{n}$ for all $n<\omega$.

Obviously, every element of a mutually Ramsey sequence $\left(\kappa_{n}\right)_{n<\omega}$ is a Ramsey cardinal. The consistency strength of the existence of a mutually Ramsey sequence $\left(\kappa_{n}\right)_{n<\omega}$ is that of one measurable cardinal. Theorem 3.2 of [4] shows:

Proposition 2.3. Let $\kappa$ be a measurable cardinal. Let $\left(\kappa_{n}\right)_{n<\omega}$ be a Prikry sequence adjoined by Prikry forcing with a measure on $\kappa$. Then there is an endsegment of $\left(\kappa_{n}\right)_{n<\omega}$ which is mutually Ramsey in the Prikry generic extension $V\left[\left(\kappa_{n}\right)_{n<\omega}\right]$.

We can now turn to the

Proof of Theorem 1.6. By the previous proposition we may generically extend the ground model to a model in which there is a sequence $\left(\kappa_{n}\right)_{n<\omega}$ of mutually Ramsey cardinals with supremum $\kappa$. Using "small forcing" we may also assume that Martin's Axiom $\mathrm{MA}_{\aleph_{1}}$ holds in the ground model $V$. We shall turn $\kappa_{0}<\kappa_{1}<\kappa_{2} \ldots<\kappa$ into $\aleph_{3}, \aleph_{5}, \aleph_{7}, \ldots, \aleph_{\omega}$ respectively by an appropriate product of Levy collapses. Set $\kappa_{-1}=\aleph_{1}$. Let

$$
P=\prod_{n<\omega} \operatorname{Col}\left(\kappa_{n-1}^{+},<\kappa_{n}\right),
$$

where $\operatorname{Col}\left(\kappa_{n-1}^{+},<\kappa_{n}\right)$ is the Levy collapse for collapsing $\kappa_{n}$ to $\kappa_{n-1}^{++}$; every condition $r \in \operatorname{Col}\left(\kappa_{n-1}^{+},<\kappa_{n}\right)$ satisfies $\operatorname{dom}(r) \subseteq \kappa_{n-1}^{+} \times \kappa_{n}$. For $p \in P$ and $n<\omega$ write $p_{n}$ instead of $p(n) . P$ is partially ordered by coordinatewise reverse inclusion:

$$
p \leqslant q \text { iff } \forall n<\omega p_{n} \supseteq q_{n} .
$$

Let $G$ be $P$-generic over $V . \quad V[G]$ is the intended generic extension, which we describe by a sequence of claims. The preservation of certain cardinals is proved by a product analysis of $P$. Let $m<\omega$ and define

$$
P_{<m}=\prod_{n<m} \operatorname{Col}\left(\kappa_{n-1}^{+},<\kappa_{n}\right) \text { and } P_{\geqslant m}=\prod_{m \leqslant n<\omega} \operatorname{Col}\left(\kappa_{n-1}^{+},<\kappa_{n}\right),
$$

both ordered by coordinatewise reverse inclusion $\leqslant$. If $p \in P$, then $p\left\lceil m \in P_{<m}\right.$ and $p\left\lceil(\omega \backslash m) \in P_{\geqslant m}\right.$. Obviously, $P_{\geqslant 0}=P$.

(1) $\left(P_{\geqslant m}, \leqslant\right)$ is $<\kappa_{m-1}^{+}$-complete.

(2) $\left(P_{<m}, \leqslant\right)$ satisfies the $\kappa_{m-1}$-chain condition.

Proof. This is obvious in case $m=0$. Let $m>0$ and let $A \subseteq P_{<m}$ with $\operatorname{card}(A)=$ $\kappa_{m-1}$. Every $p \in P_{<m}$ may be split into $p\left\lceil(m-1) \in P_{<m-1}\right.$ and $p(m-1) \in$ $\operatorname{Col}\left(\kappa_{m-2}^{+},<\kappa_{m-1}\right)$. Since $\operatorname{card}\left(P_{<m-1}\right)<\kappa_{m-1}$ we may assume that $p\lceil(m-1)$ is the same for all $p \in A$. Since $\operatorname{Col}\left(\kappa_{m-2}^{+},<\kappa_{m-1}\right)$ satisfies the $\kappa_{m-1}$-chain condition there must be $p, q \in A, p \neq q$ such that $p(m-1)$ and $q(m-1)$ are compatible in $\operatorname{Col}\left(\kappa_{m-2}^{+},<\kappa_{m-1}\right)$. Since $p \nmid(m-1)=q \uparrow(m-1), p$ and $q$ are compatible in $\left(P_{<m}, \leqslant\right)$. This proves $(2)$.

(3) Let $p \in P, p \Vdash \dot{\alpha} \in \operatorname{Ord}$. Then there is some $w \subseteq \operatorname{Ord}$, $\operatorname{card}(w)<\kappa_{m-1}$ and some $q \leqslant p$ such that

$$
q \Vdash \dot{\alpha} \in w \text { and } q\lceil m=p\lceil m \text {. }
$$


Proof. Define a sequence $\left(p^{i} \mid i<\theta\right)$ recursively such that

$$
\theta \leqslant \kappa_{m-1} \text { and } \forall i<j<\theta\left(p_{i} \leqslant p \wedge p_{j} \uparrow(\omega \backslash m) \leqslant p_{i} \uparrow(\omega \backslash m)\right) .
$$

Let $k<\kappa_{m-1}$ and let $\left(p^{i} \mid i<k\right)$ be defined satisfying the recursive condition. Define a condition $q^{k} \in P$ by

$$
q_{n}^{k}=\left\{\begin{array}{l}
p_{n}, \text { if } n<m \\
\bigcup_{i<k} p_{n}^{i}, \text { if } n \geqslant m .
\end{array}\right.
$$

$q^{k}$ is a condition by the closure properties of $P_{\geqslant m}$. If there is $p^{\prime} \leqslant q^{k}$ and some $\gamma \in$ Ord such that $p^{\prime} \Vdash \dot{\alpha}=\check{\gamma}$ and $\forall i<k p^{\prime} \uparrow m$ is incompatible with $p^{i}\lceil m$, then let $p^{k}$ be such a $p^{\prime}$ and continue. If not set $\theta=k$ and stop the recursion.

By construction, $\left(p^{i}\lceil m \mid i<\theta)\right.$ is an antichain in $P_{<m}$. Since $P_{<m}$ satisfies the $\kappa_{m-1}$-chain condition, $\theta<\kappa_{m-1}$. Define a condition $q \in P$ by

$$
q_{n}=\left\{\begin{array}{l}
p_{n}, \text { if } n<m \\
\bigcup_{i<\theta} p_{n}^{i}, \text { if } n \geqslant m .
\end{array}\right.
$$

Let $w=\left\{\gamma \in \operatorname{Ord} \mid \exists i<\theta p^{i} \Vdash \dot{\alpha}=\check{\gamma}\right\}$. Then $\operatorname{card}(w) \leqslant \theta<\kappa_{m-1}$. We show that $q \Vdash \dot{\alpha} \in w$. Note that $q=q^{\theta}$ in the above recursion. Let $r \leqslant q$. Let $s \leqslant r$ and $\delta \in$ Ord such that $s \Vdash \dot{\alpha}=\check{\delta}$. Since $p^{\theta}$ is not defined there is $i<\theta$ such that $s \uparrow m$ and $p^{i}\left\lceil m\right.$ are compatible. Then $s$ and $p^{i}$ are compatible. Let $\gamma \in w$ such that $p^{i} \Vdash \dot{\alpha}=\check{\gamma}$. By the compatibility of $s$ and $p^{i}$ we have $\delta=\gamma \in w$ and so $s \Vdash \dot{\alpha} \in \check{w}$. Thus $\{s \mid s \Vdash \dot{\alpha} \in \check{w}\}$ is dense below $q$ and $q \Vdash \dot{\alpha} \in \check{w}$. This proves (3).

(4) Let $m<\omega$. Then $\kappa_{m-1}$ and $\kappa_{m-1}^{+}$are cardinals in $V[G]$.

Proof. Assume that $p \in P$ and $p \Vdash\left(\dot{h}: \check{\theta} \rightarrow \kappa_{m-1}\right.$ is surjective $)$ for some $\theta<$ $\kappa_{m-1}$. By the previous claim we can recursively define a $\leqslant$-decreasing sequence of conditions $\left(q^{i} \mid i<\theta\right)$ and sets $w_{i} \subseteq$ Ord such that

$$
\operatorname{card}\left(w_{i}\right)<\kappa_{m-1}, q^{i} \leqslant p, q^{i} \Vdash \dot{h}(\check{i}) \in w_{i} \text { and } q^{i} \uparrow m=p\lceil m .
$$

By the $<\kappa_{m-1}^{+}$-completeness of $\left(P_{\geqslant m}, \leqslant\right)$ take $q \in P$ such that $\forall i<\theta q \leqslant q^{i}$. Let $w=\bigcup_{i<\theta} w_{i}$. Since $\kappa_{m-1}$ is regular we have $\operatorname{card}(w) \leqslant \kappa_{m-1}$ and $\sup (w)<\kappa_{m-1}$. Then $q \Vdash \operatorname{range}(\dot{h}) \subseteq w \subseteq \sup (w)<\kappa_{m-1}$, contradicting that $p$ forces $\dot{h}$ to be onto $\kappa_{m-1}$. Thus $\kappa_{m-1}$ is a cardinal in $V[G]$.

The argument for $\kappa_{m-1}^{+}$being a cardinal is virtually the same. This proves (4).

(5) Let $m<\omega$. Then no ordinal $\xi \in\left(\kappa_{m-1}^{+}, \kappa_{m}\right)$ is a cardinal in $V[G]$. This follows immediately from the basic properties of a Levy collapse.

Hence we obtain the following cardinality pattern in $V[G]$ :

(6) $\aleph_{1}^{V[G]}=\aleph_{1}^{V}, \aleph_{2}^{V[G]}=\aleph_{2}^{V}, \aleph_{3}^{V[G]}=\kappa_{0}, \aleph_{4}^{V[G]}=\kappa_{0}^{+}, \ldots, \aleph_{2 n+3}^{V[G]}=\kappa_{n}$, $\aleph_{2 n+4}^{V[G]}=\kappa_{n}^{+}, \ldots, \aleph_{\omega}^{V[G]}=\kappa$.

To prove $\operatorname{MS}\left(\aleph_{3}, \aleph_{5}, \aleph_{7}, \ldots ; \omega_{1}\right)$ in $V[G]$ consider, in $V[G]$, an $F:\left[\aleph_{\omega}\right]^{<\omega} \rightarrow \aleph_{\omega}$ and a sequence $\left(S_{n}\right)_{n<\omega}$ of sets $S_{n} \subseteq \operatorname{cof}_{\omega_{1}}$ stationary in $\kappa_{n}$. Take names $\dot{F}$ and $\dot{S}_{n}$ and a condition $p \in P$ such that

$$
p \Vdash\left(\dot{F}:\left[\aleph_{\omega}\right]^{<\omega} \rightarrow \aleph_{\omega} \text { and } \forall n<\omega\left(\dot{S}_{n} \subseteq \operatorname{cof}_{\omega_{1}} \wedge \dot{S}_{n} \text { is stationary in } \kappa_{n}\right)\right) .
$$

It suffices to "densely show" the existence of a set $X \subseteq \aleph_{\omega}$ such that

$$
\forall n<\omega \sup \left(\left(F^{\prime \prime}[X]^{<\omega}\right) \cap \aleph_{2 n+3}\right) \leqslant \sup \left(X \cap \aleph_{2 n+3}\right) \in S_{n} .
$$


We shall fix the values $\dot{F}(x)$ for a large set of $x \in[\kappa]^{<\omega}$. We shall define conditions $p(x) \in P$ such that $p(x) \Vdash \dot{F}(x)=\check{\alpha}$ for some ordinal $\alpha<\kappa$. The recursive definition of the function $p(x)$ will proceed along the well-ordering $<^{*}$ of $\left.[\mathrm{Ord}]\right]^{<\omega}$ by largest differences:

$$
x<^{*} y \text { iff } \max (x \triangle y) \in y .
$$

Let $t$ be a type (relative to the sequence $\left.\kappa_{0}<\kappa_{1}<\ldots<\kappa\right)$. A sequence $(p(x) \mid x \in$ $[\kappa]^{t}$ ) is called partially constant (p.c.) (below $p$ ) if the following two conditions are satisfied:

(7) $\forall x \in[\kappa]^{t} p(x) \leqslant p$;

(8) $\forall x, y \in[\kappa]^{t} \forall n<\omega\left(x \backslash \kappa_{n-1}=y \backslash \kappa_{n-1} \rightarrow p_{n}(x)=p_{n}(y)\right)$.

By (8), the $n$-th component of $p(x)$ is determined by $x \backslash \kappa_{n-1}$. Later we shall use mutual indiscernibility to make $p_{n}(x)$ only dependent on $x \cap\left(\kappa_{n-1}, \kappa_{n}\right)$.

We shall construct a countable family of partially constant sequences which decide certain values of $\dot{F}$ and which witness some compatibilities. Note that the constant sequence $p(x) \equiv p$ is partially constant. The following two extension properties yield new partially constant sequences starting from given ones.

(9) Let $t, \bar{t}$ be types and $a \subseteq \omega$ such that for all $z \in[\kappa]^{t}$ type $(z\lceil a)=\bar{t}$ holds. Let $\left(p(x) \mid x \in[\kappa]^{\bar{t}}\right)$ be a p.c. sequence. Define $\left(q(z) \mid z \in[\kappa]^{t}\right)$ by $q_{n}(z)=p_{n}(z\lceil a)$. Then $\left(q(z) \mid z \in[\kappa]^{t}\right)$ is p.c.

Proof. Let $x, y \in[\kappa]^{t}$ and $x \backslash \kappa_{n-1}=y \backslash \kappa_{n-1}$. Then $\left(x\lceil a) \backslash \kappa_{n-1}=\left(y\lceil a) \backslash \kappa_{n-1}\right.\right.$ and

which proves (9).

$$
q_{n}(x)=p_{n}\left(x\lceil a)=p_{n}\left(y\lceil a)=q_{n}(y)\right.\right.
$$

(10) Let $t$ be a type and let $\left(p(x) \mid x \in[\kappa]^{t}\right)$ be a p.c. sequence. Then there is a p.c. sequence $\left(q(x) \mid x \in[\kappa]^{t}\right)$ and a sequence $\left(v(z) \mid z \in[\kappa]^{t}\right)$ of finite sets of ordinals $<\kappa$ such that

$$
\forall x \in[\kappa]^{t}(q(x) \leqslant p(x) \text { and } \forall z \subseteq x \exists \alpha \in v(x) q(x) \Vdash \dot{F}(z)=\check{\alpha}) .
$$

Proof. Define sequences $\left(r(x) \mid x \in[\kappa]^{t}\right)$ and $\left(v(x) \mid x \in[\kappa]^{t}\right)$ by simultaneous induction on $<^{*}$ such that the following two properties hold:

(11) $r(x) \leqslant p(x)$;

(12) $\forall u, x \in[\kappa]^{t}, u<^{*} x \forall n<\omega\left(u \backslash \kappa_{n-1}=x \backslash \kappa_{n-1} \rightarrow r_{n}(u) \geqslant r_{n}(x)\right)$.

Let $x \in[\kappa]^{t}$ and let $r(u), v(u)$ be adequately defined for $u<^{*} x, x \in[\kappa]^{t}$. Then define $\bar{r} \in P$ by

$$
\bar{r}_{n}=p_{n}(x) \cup \bigcup\left\{r_{n}(u) \mid u<^{*} x, u \backslash \kappa_{n-1}=x \backslash \kappa_{n-1}\right\} .
$$

$\bar{r}$ is a properly defined condition in $P$ by the growth condition (12) and the closure conditions of the Levy collapses. Also $\bar{r} \leqslant p(x)$. Then choose $r(x) \leqslant \bar{r}$ and $v(x) \in[\kappa]^{<\omega}$ such that

$$
\forall z \subseteq x \exists \alpha \in v(x) r(x) \Vdash \dot{F}(z)=\check{\alpha} .
$$

Note that the recursive condition (12) is satisfied at $x$ : if $u \in[\kappa]^{t}, u<^{*} x$ and $u \backslash \kappa_{n-1}=x \backslash \kappa_{n-1}$, then $r_{n}(x) \leqslant \bar{r}_{n} \leqslant r_{n}(u)$.

Finally define the sequence $\left(q(x) \mid x \in[\kappa]^{t}\right)$ by

$$
q_{n}(x)=p_{n}(x) \cup \bigcup\left\{r_{n}(u) \mid u \in[\kappa]^{t}, u \backslash \kappa_{n-1}=x \backslash \kappa_{n-1}\right\} .
$$

$(q(x))$ is p.c. by construction and satisfies claim (10). 
(13) Let $t, \bar{t}$ be types and $a_{0}, a_{1} \subseteq \omega$ be such that for all $y \in[\kappa]^{t}$ : type $\left(y\left\lceil a_{0}\right)=\right.$ type $\left(y\left\lceil a_{1}\right)=\bar{t}\right.$. Let $\left(p(x) \mid x \in[\kappa]^{\bar{t}}\right)$ be a p.c. sequence. Then there is a p.c. sequence $\left(q(y) \mid y \in[\kappa]^{t}\right)$ such that for all $y \in[\kappa]^{t}$, if $p\left(y\left\lceil a_{0}\right)\right.$ and $p\left(y\left\lceil a_{1}\right)\right.$ are compatible in $P$, then

$$
q(y) \leqslant p\left(y\left\lceil a_{0}\right) \text { and } q(y) \leqslant p\left(y\left\lceil a_{1}\right) .\right.\right.
$$

Proof. For $y \in[\kappa]^{t}$ define $q(y)$ by $q_{n}(y)=p_{n}\left(y\left\lceil a_{0}\right) \cup p_{n}\left(y\left\lceil a_{1}\right)\right.\right.$ if the right-hand side is a condition in $\operatorname{Col}\left(\kappa_{n-1}^{+},<\kappa_{n}\right)$, and $q_{n}(y)=p_{n}\left(y\left\lceil a_{0}\right)\right.$ otherwise.

We only have to check that $(q)$ is p.c. Let $x, y \in[\kappa]^{t}$ and $x \backslash \kappa_{n-1}=y \backslash \kappa_{n-1}$. By definition

$$
q(x) \leqslant p\left(x\left\lceil a_{0}\right) \leqslant p .\right.
$$

$x \backslash \kappa_{n-1}=y \backslash \kappa_{n-1}$ implies that $\left(x \mid a_{0}\right) \backslash \kappa_{n-1}=\left(y\left\lceil a_{0}\right) \backslash \kappa_{n-1}\right.$ and $\left(x \nmid a_{1}\right) \backslash \kappa_{n-1}=\left(y\left\lceil a_{1}\right) \backslash \kappa_{n-1}\right.$. Since $(p)$ is p.c., $p_{n}\left(x \uparrow a_{0}\right)=p_{n}\left(y\left\lceil a_{0}\right)\right.$ and $p_{n}\left(x\left\lceil a_{1}\right)=p_{n}\left(y\left\lceil a_{1}\right)\right.\right.$.

Case 1. $p_{n}\left(x\left\lceil a_{0}\right), p_{n}\left(x\left\lceil a_{1}\right)\right.\right.$ are compatible in $\operatorname{Col}\left(\kappa_{i-1}^{+},<\kappa_{i}\right)$. Then

$$
q_{n}(x)=p_{n}\left(x \lceil a _ { 0 } ) \cup p _ { n } \left(x\left\lceil a_{1}\right)=p_{n}\left(y \lceil a _ { 0 } ) \cup p _ { n } \left(y\left\lceil a_{1}\right)=q_{n}(y) .\right.\right.\right.\right.
$$

Case 2. $p_{n}\left(x\left\lceil a_{0}\right), p_{n}\left(x\left\lceil a_{1}\right)\right.\right.$ are incompatible in $\operatorname{Col}\left(\kappa_{i-1}^{+},<\kappa_{i}\right)$. Then

$$
q_{n}(x)=p_{n}\left(x\left\lceil a_{0}\right)=p_{n}\left(y\left\lceil a_{0}\right)=q_{n}(y) .\right.\right.
$$

This proves (13).

Properties (9), (10) and (13) are construction principles for p.c. sequences $q(x)$ from p.c. sequences $p(x)$. By an appropriate organisation one can construct a sequence of p.c. sequences

$$
p^{0}, p^{1}, v^{1}, p^{2}, v^{2}, \ldots
$$

such that $p^{0}$ is the constant sequence $p^{0}(x) \equiv p$ and such that for any given $p^{k}$ and any constellation of types as in (9), (10) or (13) there are sequences $p^{l}$ and possibly $v^{l}$ which relate to $p^{k}$ as $q$ and $v$ relate to $p$ in (9), (10) or (13) respectively. We may also assume that every $p^{k}, v^{k}$ is definable over some common sufficiently large structure

$$
\left(V_{\theta}, \in, \ldots, \dot{F}, \dot{S}_{0}, \dot{S}_{1}, \ldots, p\right) \text {, where } \theta \supseteq \kappa .
$$

By the mutual Ramseyness of $\kappa_{0}, \kappa_{1}, \ldots$ take a mutually homogeneous system $I_{0}, I_{1}, \ldots$ for the structure $\left(V_{\theta}, \in, \ldots, \dot{F}, \dot{S}_{0}, \dot{S}_{1}, \ldots, p\right)$ such that $I_{i} \subseteq \kappa_{i}$ and such that $\operatorname{card}\left(I_{i}\right)=\kappa_{i}$. One can check that each of these indiscernibles is inaccessible.

Consider any sequence $I_{0}^{\prime}, I_{1}^{\prime}, \ldots$ such that each $I_{i}^{\prime}$ is a subset of $I_{i}$ of order type $\omega_{1}$. We shall later choose such a sequence appropriately for the mutual stationarity property. We view $I^{\prime}=\bigcup_{i<\omega} I_{i}^{\prime}$ as a system of generating indiscernibles for a substructure of $\left(V_{\theta}, \in, \ldots, \dot{F}, \dot{S}_{0}, \dot{S}_{1}, \ldots, p\right)$. Let

$$
P^{\prime}=\left\{p^{k}(z) \mid z \subseteq I^{\prime}, k<\omega\right\} \subseteq P .
$$

We intend to apply $\mathrm{MA}_{\aleph_{1}}$ to $P^{\prime}$ to produce a sufficiently generic condition in $P$. This requires the countable chain condition and certain densities.

$(14)\left(P^{\prime}, \leqslant\right)$ satisfies the countable chain condition.

Proof. Let $A \subseteq P^{\prime}$ be uncountable. By possibly thinning out $A$ we may assume that there is some $k<\omega$ such that every $q \in A$ is of the form $q=p^{k}(z)$ for some $z \subseteq I^{\prime}$, i.e.,

$$
A=\left\{p^{k}(z) \mid z \in Z\right\}
$$


where $Z \subseteq\left[I^{\prime}\right]^{t}$ is uncountable and $t$ is the appropriate argument type for $p^{k}$. By a $\Delta$-system argument one can assume that $Z$ is an uncountable $\Delta$-system of finite sets with kernel $a$ :

$$
\forall z \in Z: a \subseteq z \text { and } \forall z, z^{\prime} \in Z, z \neq z^{\prime}:(z \backslash a) \cap\left(z^{\prime} \backslash a\right)=\emptyset .
$$

Since the set $\bigcup_{n<\omega}\left[I^{\prime} \cap\left(\kappa_{n-1}, \max \left(a \cap\left(\kappa_{n-1}, \kappa_{n}\right)\right)\right)\right]$ is countable there is an uncountable $Z^{\prime} \subseteq Z$ such that

$$
\forall z \in Z^{\prime} \forall n<\omega: \max \left(a \cap\left(\kappa_{n-1}, \kappa_{n}\right)\right)<\min \left((z \backslash a) \cap\left(\kappa_{n-1}, \kappa_{n}\right)\right) .
$$

Choose $x \in Z^{\prime}$. Again, there is an uncountable $Z^{\prime \prime} \subseteq Z^{\prime}$ such that

$$
\forall z \in Z^{\prime \prime} \forall n<\omega: \max \left(x \cap\left(\kappa_{n-1}, \kappa_{n}\right)\right)<\min \left((z \backslash a) \cap\left(\kappa_{n-1}, \kappa_{n}\right)\right) .
$$

Choose $y \in Z^{\prime \prime}$. We shall show that $p^{k}(x)$ and $p^{k}(y)$ are compatible in $P$. Let $n<\omega$. It suffices to show that $p_{n}^{k}(x)$ and $p_{n}^{k}(y)$ are compatible in $\operatorname{Col}\left(\kappa_{n-1}^{+},<\kappa_{n}\right)$. Since $p^{k}$ is p.c. we have

$$
p_{n}^{k}(y)=p_{n}^{k}\left(\left(x \cap \kappa_{n-1}\right) \cup\left(y \cap\left(\kappa_{n-1}, \kappa_{n}\right)\right)\right),
$$

i.e., we can assume for the compatibility argument that $x \cap \kappa_{n-1}=y \cap \kappa_{n-1}$.

Case 1. $(x \backslash a) \cap\left(\kappa_{n-1}, \kappa_{n}\right)=\emptyset$, i.e., $x \cap\left(\kappa_{n-1}, \kappa_{n}\right)=a \cap\left(\kappa_{n-1}, \kappa_{n}\right)$. Since $x$ and $y$ have the same type, also $y \cap\left(\kappa_{n-1}, \kappa_{n}\right)=a \cap\left(\kappa_{n-1}, \kappa_{n}\right)$. The term $p_{n}^{k}(x)$ is an element of $V_{\kappa_{n}}$ and can thus be viewed as a term of the structure $\left(V_{\theta}, \in, \ldots, \dot{F}, \dot{S}_{0}, \dot{S}_{1}, \ldots, p\right)$ mapping $x$ to an ordinal $<\kappa_{n}$. Similarly, $p_{n}^{k}(y)$ can be viewed as the same term mapping $y$ to an ordinal $<\kappa_{n}$. Since $x \cap \kappa_{n}=y \cap \kappa_{n}$ the mutual homogeneity of the system $I$ implies that

$$
p_{n}^{k}(x)=p_{n}^{k}(y)
$$

hence both sides are trivially compatible in $\operatorname{Col}\left(\kappa_{n-1}^{+},<\kappa_{n}\right)$.

Case 2. $(x \backslash a) \cap\left(\kappa_{n-1}, \kappa_{n}\right) \neq \emptyset$. Then let $\beta=\min \left((x \backslash a) \cap\left(\kappa_{n-1}, \kappa_{n}\right)\right)$ and $\gamma=\min \left((y \backslash a) \cap\left(\kappa_{n-1}, \kappa_{n}\right)\right)$. As in Case 1, the terms $p_{n}^{k}(x) \uparrow\left(\kappa_{n-1}^{+} \times \beta\right)$ and $p_{n}^{k}(y) \uparrow\left(\kappa_{n-1}^{+} \times \gamma\right)$ can be viewed as ordinals $<\beta$ and $<\gamma$ respectively. Since $x \cap \beta=y \cap \beta=y \cap \gamma$ the mutual homogeneity of the sytem $I$ implies

$$
p_{n}^{k}(x) \uparrow\left(\kappa_{n-1}^{+} \times \beta\right)=p_{n}^{k}(y) \uparrow\left(\kappa_{n-1}^{+} \times \gamma\right)
$$

and

$$
\operatorname{dom}\left(p_{n}^{k}(x)\right) \subseteq \kappa_{n-1}^{+} \times \gamma .
$$

Hence $p_{n}^{k}(x)$ and $p_{n}^{k}(y)$ are compatible in $\operatorname{Col}\left(\kappa_{n-1}^{+},<\kappa_{n}\right)$.

So in both cases, we obtain compatibility of $p_{n}^{k}(x)$ and $p_{n}^{k}(y)$ for all $n<\omega$; hence $p^{k}(x)$ and $p^{k}(y)$ are compatible in $P$. By construction of the sequence $p^{0}, p^{1}, v^{1}, p^{2}, v^{2}, \ldots P^{\prime}$ contains a compatibility element for $p^{k}(x)$ and $p^{k}(y)$, and so $p^{k}(x)$ and $p^{k}(y)$ are compatible in $P^{\prime}$. Thus no uncountable subset of $P^{\prime}$ is an antichain in $P^{\prime}$, which proves (14).

Let $W=\bigcup\left\{v^{k}(z) \mid z \subseteq I^{\prime}, k<\omega\right\}$ be the set of possible values $\dot{F}(z)$ on the indiscernibles $I^{\prime}$.

(15) Let $y \subseteq I^{\prime}, y$ finite. Then the set

$$
D_{y}=\left\{r \in P^{\prime} \mid \exists \alpha \in W r \Vdash_{P} \dot{F}(y)=\check{a}\right\}
$$

is dense in $P^{\prime}$. Observe that the forcing $\Vdash_{P}$ in the definition of $D_{y}$ is done with respect to the full partial order $P$. 
Proof. Let $p^{k}(x) \in P^{\prime}, x \subseteq I^{\prime}$. Let $z=x \cup y \subseteq I^{\prime}$. By the construction of the sequence $p^{0}, p^{1}, v^{1}, p^{2}, v^{2}, \ldots$ according to property (9) there is $l<\omega$ such that $p^{k}(x)=p^{l}(z)$. Furthermore, according to (9) there is some $m<\omega$ and some finite $z \subseteq I^{\prime}$ such that $x, y \subseteq z$ and

$$
p^{l}(z) \leqslant p^{k}(x) \text { and } p^{l}(z) \Vdash \dot{F}(y)=v^{l}(z) \in \check{W} .
$$

This proves (15).

By $\mathrm{MA}_{\aleph_{1}}$ take a generic filter $H^{\prime} \subseteq P^{\prime}$ for the family $\left\{D_{y} \mid y \subseteq I^{\prime}, y\right.$ finite $\}$. Define a condition $p^{\prime} \in P$ by $p_{n}^{\prime}=\bigcup\left\{r_{n} \mid r \in H^{\prime}\right\}$. Observe that all $r_{n}$ for $r \in H^{\prime}$ are compatible by the genericity of $H^{\prime}$. Also $p^{\prime} \leqslant p$.

(16) $p^{\prime} \Vdash \dot{F}^{\prime \prime}\left[I^{\prime}\right]<\omega \subseteq \breve{W}$.

Proof. Let $y \in I^{\prime}, y$ finite. By the genericity of $H^{\prime}$ there is $r \in H^{\prime} \cap D_{y}$. Take $\alpha \in W$ such that $r \Vdash \dot{F}(y)=\check{\alpha}$. Then $r \Vdash \dot{F}(y) \in \check{W}$ and $p^{\prime} \leqslant r$. This proves (16).

(17) $\forall n<\omega, \sup \left(W \cap \kappa_{n}\right) \leqslant \sup I_{n}^{\prime}$.

Proof. Let $\alpha=v^{k}(z) \in W \cap \kappa_{n}, z \in I^{\prime}$. Since $I_{n}$ is cofinal in $\kappa_{n}$ there is some $\zeta \in I_{n}$ such that $\alpha<\zeta<\kappa_{n}$. Since $I_{n}^{\prime}$ has limit order type there is $\zeta^{\prime} \in I_{n}^{\prime}$ such that $\max \left(z \cap \kappa_{n}\right)<\zeta^{\prime}$. By the indiscernibility properties $\alpha=v^{k}(z)<\zeta$ implies $\alpha=v^{k}(z)<\zeta^{\prime}<\sup I_{n}^{\prime}$, proving (17).

In the following we shall isomorphically shift the indiscernible sets $I_{0}^{\prime}, I_{1}^{\prime}, \ldots$ to some other such sets $I_{0}^{\prime \prime}, I_{1}^{\prime \prime}, \ldots$ so that the stationarity property $\sup I_{n}^{\prime \prime} \in \dot{S}_{n}$ is also forced. If $I_{0}^{\prime \prime}, I_{1}^{\prime \prime}, \ldots$ is another sequence such that each $I_{i}^{\prime \prime}$ is a subset of $I_{i}$ of order type $\omega_{1}$, set $I^{\prime \prime}=\bigcup_{i<\omega} I_{i}^{\prime \prime}$. These sets of generating indiscernibles are orderisomorphic by some isomorphism $\pi: I^{\prime} \cong I^{\prime \prime}$. The isomorphism can canonically be extended to an isomorphism $\tilde{\pi}$ of the substructures of $\left(V_{\theta}, \in, \ldots, \dot{F}, \dot{S}_{0}, \dot{S}_{1}, \ldots, p\right)$ generated by the indiscernible sets $I^{\prime}$ and $I^{\prime \prime}$ respectively: $\tilde{\pi}$ is defined by $t(z) \mapsto$ $t\left(\pi^{\prime \prime} z\right)$ for Skolem terms $t$ of $\left(V_{\theta}, \in, \ldots, \dot{F}, \dot{S}_{0}, \dot{S}_{1}, \ldots, p\right)$ and $z \subseteq I^{\prime}$. In particular, $\tilde{\pi}$ preserves all components of the previous construction.

Let $P^{\prime \prime}=\left\{p^{k}(z) \mid z \subseteq I^{\prime \prime}, k<\omega\right\} \subseteq P$. Then $\tilde{\pi}\left\lceil P^{\prime}:\left(P^{\prime}, \leqslant\right) \cong\left(P^{\prime \prime}, \leqslant\right)\right.$ is a well-defined isomorphism. $\tilde{\pi} \mid W: W \cong W^{\prime \prime}=\left\{v^{k}(z) \mid z \subseteq I^{\prime \prime}, k<\omega\right\}$ takes the sets of possible values into each other. For each $y \subseteq I^{\prime}, y$ finite, the set $D_{y}^{\prime \prime}=\tilde{\pi}^{\prime \prime} D_{y}$ satisfies

$$
D_{y}^{\prime \prime}=\left\{r \in P^{\prime \prime} \mid \exists \alpha \in W^{\prime \prime} r \Vdash_{P} \dot{F}(y)=\check{a}\right\} .
$$

The set $H^{\prime \prime}=\tilde{\pi}^{\prime \prime} H^{\prime}$ is generic in $P^{\prime \prime}$ for the family $\left\{D_{y}^{\prime \prime} \mid y \subseteq I^{\prime \prime}, y\right.$ finite $\}$. Then the condition $p^{\prime \prime}$ defined by $p_{n}^{\prime \prime}=\bigcup\left\{r_{n} \mid r \in H^{\prime \prime}\right\}$ satisfies the analogue of (16): $p^{\prime \prime} \Vdash \dot{F}^{\prime \prime}\left[I^{\prime \prime}\right]^{<\omega} \subseteq \check{W}^{\prime \prime}$. Finally the analogue of (17) holds: $\forall n<\omega \sup \left(W^{\prime \prime} \cap \kappa_{n}\right) \leqslant$ $\sup I_{n}^{\prime \prime}$.

We show that the domains of the condition $p^{\prime}$ are closely positioned around the generating indiscernibles in $I^{\prime}$.

(18) Let $r=p^{k}(z), z \subseteq I^{\prime}$, and $n<\omega$. Let $\xi \in I_{n} \backslash I_{n}^{\prime}$. Then

$$
\operatorname{dom} p_{n}^{k}(z) \cap \kappa_{n-1}^{+} \times\left[\xi, \min \left(I_{n} \backslash(\xi+1)\right)\right)=\emptyset .
$$

Proof. Assume not. Let $\xi^{\prime}=\min \left(I_{n} \backslash(\xi+1)\right)$ and

$$
s\left(z, \xi^{\prime}\right)=\sup \left\{\beta<\xi^{\prime} \mid \exists \alpha<\kappa_{n-1}^{+}(\alpha, \beta) \in \operatorname{dom} p_{n}^{k}(z)\right\} .
$$


Since $\operatorname{card}\left(p_{n}^{k}\right) \leqslant \kappa_{n-1}$ and by the inaccessibility of the indiscernibles,

$$
\xi \leqslant s\left(z, \xi^{\prime}\right)<\xi^{\prime}
$$

Let $\xi^{\prime \prime}=\min \left(I_{n} \backslash\left(\xi^{\prime}+1\right)\right)$. Let $z^{\prime \prime} \subseteq I_{n}$ with type $\left(z^{\prime \prime}\right)=\operatorname{type}(z), z^{\prime \prime} \cap \xi^{\prime}=z \cap \xi^{\prime}$ and

$$
\xi^{\prime} \in z \rightarrow \xi^{\prime \prime}=\min \left(z^{\prime \prime} \backslash \xi^{\prime}\right) \text { and } \xi^{\prime} \notin z \rightarrow \xi^{\prime \prime}<\min \left(z^{\prime \prime} \backslash \xi^{\prime}\right) .
$$

By indiscernibility, $s\left(z^{\prime \prime}, \xi^{\prime \prime}\right)=s\left(z, \xi^{\prime}\right)$ and so $s\left(z^{\prime \prime}, \xi^{\prime \prime}\right)<\xi^{\prime}$. Again by indiscernibility, observing that $\xi \notin z: s\left(z, \xi^{\prime}\right)<\xi$. But this contradicts $\xi \leqslant s\left(z, \xi^{\prime}\right)$, proving (18).

Since the condition $p^{\prime}$ is a union of conditions $p_{n}^{k}(z)$ as in (18) it follows that:

(19) Let $n<\omega$ and $\xi \in I_{n} \backslash I_{n}^{\prime}$. Then

$$
\operatorname{dom} p_{n}^{\prime} \cap \kappa_{n-1}^{+} \times\left[\xi, \min \left(I_{n} \backslash(\xi+1)\right)\right)=\emptyset
$$

and hence

$$
\operatorname{dom} p_{n}^{\prime} \subseteq \kappa_{n-1}^{+} \times\left(\min I_{n} \cup \bigcup_{\xi \in I_{n}^{\prime}}\left[\xi, \min \left(I_{n} \backslash(\xi+1)\right)\right)\right) .
$$

Define $\bar{p} \in P$ by

$$
\bar{p}_{n}=p_{n}^{\prime} \uparrow\left(\kappa_{n-1}^{+} \times \min I_{n}\right) .
$$

Since the elements of $I^{\prime}$ are indiscernible with respect to the constant $p$ we have that $p_{n}$ is "below" the minimum of $I_{n}$. Hence $\bar{p} \leqslant p$. The above isomorphism $\pi: I^{\prime} \cong I^{\prime \prime}$ of indiscernibles also shows that for any $I^{\prime \prime}$ as above we obtain:

(20) $\bar{p}_{n}=p_{n}^{\prime \prime} \uparrow\left(\kappa_{n-1}^{+} \times \min I_{n}\right)$.

Consider a $P$-generic filter $\bar{G} \ni \bar{p}$ over $V$. In $V[\bar{G}], \bar{S}_{n}=\dot{S}_{n}^{\bar{G}} \subseteq \kappa_{n}$ is stationary in $\kappa_{n}$ and $\bar{S}_{n} \subseteq \operatorname{cof}_{\omega_{1}}$. We need elements of $\bar{S}_{n}$ which are high-level limits of indiscernibles. It is easy to see that:

(21) For all $n<\omega$ the set $I_{n}^{*}=\left\{\beta<\kappa_{n} \mid \forall \delta<\beta\right.$, otp $\left.\left(I_{n} \cap(\delta, \beta)\right)>\kappa_{n-1}^{+}\right\}$is closed unbounded in $\kappa_{n}$.

Take $\beta_{n} \in I_{n}^{*} \cap \bar{S}_{n}$. Since the forcing $P$ is $<\aleph_{2}$-complete, $\left(\beta_{n} \mid n<\omega\right) \in V$ and every $\beta_{n}$ has cofinality $\omega_{1}$ in $V$. Let $q \leqslant \bar{p}$ such that $q \Vdash \forall n<\omega \beta_{n} \in \dot{S}_{n}$.

The following argument takes place in the ground model $V$. For each $n<\omega$ choose a strictly increasing sequence $\left(\beta_{n}^{i} \mid i<\omega_{1}\right)$ converging to $\beta_{n}$ such that

$$
\forall i<\omega_{1} \operatorname{card}\left(I_{n} \cap\left(\beta_{n}^{i}, \beta_{n}^{i+1}\right)\right) \geqslant \kappa_{n-1}^{+} .
$$

Since $\operatorname{card}\left(q_{n}\right)<\kappa_{n-1}^{+}$there exists $\xi_{n}^{i} \in I_{n} \cap\left(\beta_{n}^{i}, \beta_{n}^{i+1}\right)$ such that:

(22) $\operatorname{dom} q_{n} \cap \kappa_{n-1}^{+} \times\left[\xi_{n}^{i}, \min \left(I_{n} \backslash\left(\xi_{n}^{i}+1\right)\right)\right)=\emptyset$.

We can now define appropriate sets $I_{n}^{\prime \prime}$ of indiscernibles for the mutual stationarity property. Let $I_{n}^{\prime \prime}=\left\{\xi_{n}^{i} \mid i<\omega_{1}\right\}$ and $I^{\prime \prime}=\bigcup_{n<\omega} I_{n}^{\prime \prime}$. Define $p^{\prime \prime}$ and $W^{\prime \prime}$ from $I^{\prime \prime}$ as above. Then

$$
p^{\prime \prime} \Vdash \dot{F}^{\prime \prime}\left[I^{\prime \prime}\right]^{<\omega} \subseteq \check{W}^{\prime \prime} .
$$

(23) The conditions $q$ and $p^{\prime \prime}$ are compatible in $P$.

Proof. Let $n<\omega$. By (19) transferred to $I^{\prime \prime}$ we have

$$
\operatorname{dom} p_{n}^{\prime \prime} \subseteq \kappa_{n-1}^{+} \times\left(\min I_{n} \cup \bigcup_{\xi \in I_{n}^{\prime \prime}}\left[\xi, \min \left(I_{n} \backslash(\xi+1)\right)\right)\right) .
$$

By $(20), p_{n}^{\prime \prime} \uparrow\left(\kappa_{n-1}^{+} \times \min I_{n}\right)=\bar{p}_{n} \geqslant q_{n}$. Hence $q$ and $p^{\prime \prime}$ are compatible on the rectangle $\kappa_{n-1}^{+} \times \min I_{n}$. By $(22)$, the other rectangles $\kappa_{n-1}^{+} \times\left[\xi_{n}^{i}, \min \left(I_{n} \backslash\left(\xi_{n}^{i}+1\right)\right)\right)$ 
where $p_{n}^{\prime \prime}$ might be defined do not intersect the domain of $q_{n}$. So there is no conflict between $q_{n}$ and $p_{n}^{\prime \prime}$. This proves (23).

Let $r \leqslant q, p^{\prime \prime}$. By the analogues of (16) and (17) for $I^{\prime \prime}$,

$$
r \Vdash \forall n<\omega \sup \left(\left(\dot{F}^{\prime \prime}\left[I^{\prime \prime}\right]^{<\omega}\right) \cap \aleph_{2 n+3}\right) \leqslant \sup \left(I^{\prime \prime} \cap \aleph_{2 n+3}\right)=\check{\beta}_{n} \in \dot{S}_{n} .
$$

So one can densely force the mutual stationarity of $\left(\dot{S}_{n} \mid n<\omega\right)$, i.e., the mutual stationarity property $\mathrm{MS}\left(\aleph_{3}, \aleph_{5}, \aleph_{7}, \ldots ; \omega_{1}\right)$ holds in $V[G]$.

\section{VARIANTS AND DISCUSSION}

The principle $\mathrm{MS}(\ldots)$ allows many variations of parameters. We shall discuss some typical instances which are also true in the above or similar forcing extensions.

In the construction a mutual stationarity substructure of size $\aleph_{1}$ is constructed from $\aleph_{1}$ indiscernibles using $\mathrm{MA}_{\aleph_{1}}$. The indiscernibles, among other things, ensure that the supremum of the substructure below certain cardinals lies in a given stationary set. The point of the previous construction was that this is possible if the stationary set consists of ordinals of cofinality $\omega_{1}$ but the argument goes through a fortiori for ordinals of countable cofinality. So one may freely alternate between the cofinalities $\omega_{1}$ and $\omega$ to obtain

Theorem 3.1. Let $\kappa$ be a measurable cardinal. Then in the forcing extension of the main Theorem 1.6, $\mathrm{MS}\left(\aleph_{3}, \aleph_{5}, \aleph_{7}, \ldots ;\left(\lambda_{n}\right)_{n<\omega}\right)$ holds for every sequence $\left(\lambda_{n}\right)_{n<\omega}$ with $\forall n<\omega \lambda_{n} \in\left\{\omega, \omega_{1}\right\}$. E.g., $\operatorname{MS}\left(\aleph_{3}, \aleph_{5}, \aleph_{7}, \aleph_{9}, \ldots ; \omega_{1}, \omega_{0}, \omega_{1}, \omega_{0}, \ldots\right)$ is true in the extension.

For the proof of Theorem 1.7 consider the suprema $\gamma_{n}=\sup \left(\left(F^{\prime \prime}\left[I^{\prime \prime}\right]<\omega\right) \cap \aleph_{2 n+2}\right)$ of the mutual stationary substructure below the cardinals $\aleph_{2}, \aleph_{4}, \ldots$. Since $\aleph_{2 n+2}$ is a regular cardinal we may assume that the structure

$$
\left(V_{\theta}, \in, \ldots, \dot{F}, \dot{S}_{0}, \dot{S}_{1}, \ldots, p\right)
$$

contains functions $w^{l}$ which code suprema of the value function $v^{k}$ below $\aleph_{2 n+2}$ : if $\operatorname{dom}\left(v^{k}\right)=[\kappa]^{t}$, then

$$
\forall z \in[\kappa]^{t}\left(v^{k}(z)<\aleph_{2 n+2} \rightarrow v^{k}(z)<w^{l}\left(z \backslash \aleph_{2 n+1}\right)\right) .
$$

We claim that then $\operatorname{cof}\left(\gamma_{n}\right)=\omega$. By claim (15) of the main proof, every element $\xi \in F^{\prime \prime}\left[I^{\prime \prime}\right]^{<\omega}$ is of the form $\xi=v^{k}(z)$ for some $z \subseteq I^{\prime \prime}$ and $k<\omega$. By (3.1), $\xi=v^{k}(z)<w^{l}\left(z \backslash \aleph_{2 n+1}\right)$. Since $z \backslash \aleph_{2 n+1}$ are indiscernibles for values $<\aleph_{2 n+2}$ the countable set

$$
\left\{w^{l}(x) \mid x \subseteq I^{\prime \prime} \backslash \aleph_{2 n+2}\right\}
$$

bounds every element of $\left(F^{\prime \prime}\left[I^{\prime \prime}\right]^{<\omega}\right) \cap \aleph_{2 n+2}$. On the other hand, we may also assume that all the values in $\left\{w^{l}(x) \mid x \subseteq I^{\prime \prime} \backslash \aleph_{2 n+2}\right\}$ are realised as values in $F^{\prime \prime}\left[I^{\prime \prime}\right]^{<\omega}$. Hence $\operatorname{cof}\left(\gamma_{n}\right)=\omega$. This means that in the generic extension we can find mutually generic substructures whose suprema below the even $\aleph_{2 n+2}$ have cofinality $\omega$ and below the odd $\aleph_{2 n+3}$ have cofinality $\omega_{1}$. This proves Theorem 1.7.

In view of this theorem it is natural to ask whether even the mutual stationarity property $\operatorname{MS}\left(\aleph_{2}, \aleph_{3}, \aleph_{4}, \aleph_{5}, \ldots ; \omega_{0}, \omega_{1}, \omega_{0}, \omega_{1}, \ldots\right)$ holds in the model. By the methods of [5] one can however show that the consistency strength of that property is strictly larger than the existence of one measurable cardinal.

The combinatorics of our forcing construction will also go through for product forcings of the form $Q=\prod_{n<\omega} \operatorname{Col}\left(\lambda_{n},<\kappa_{n}\right)$ where each $\lambda_{n}$ is a regular cardinal in 
the interval $\left[\kappa_{n-1}^{+}, \kappa_{n}\right)$. So from one measurable cardinal, one can force properties such as

$$
\operatorname{MS}\left(\aleph_{4}, \aleph_{9}, \aleph_{16}, \aleph_{25}, \ldots ; \omega_{1}\right) \text { or } \operatorname{MS}\left(\aleph_{\omega+2}, \aleph_{\omega \cdot 2+2}, \aleph_{\omega \cdot 3+2}, \ldots ; \omega_{1}\right)
$$

\section{REFERENCES}

1. M. Foreman and M. Magidor. Mutually stationary sequences of sets and the non-saturation of the non-stationary ideal on $P_{\kappa}(\lambda)$. Acta Math., 186:271-300, 2001. MR.1846032 (2002g:03094)

2. M. Foreman, J. Cummings and M. Magidor. Canonical structure in the universe of set theory: part two. Annals of Pure and Applied Logic, 142:55-75, 2006.

3. A. Kanamori and M. Magidor. The evolution of large cardinal axioms in set theory. In G. H. Müller and D. S. Scott, editors, Higher Set Theory, number 669 in Lecture Notes in Math., pages 99-275. Springer-Verlag. MR0520190 (80b:03083)

4. P. Koepke. The consistency strength of the free-subset property for $\omega_{\omega}$. J. Symbolic Logic, 49:1198-1204, 1984. MR0771788 (86f:03091)

5. P. Koepke and P. Welch. On the strength of mutual stationarity. In Joan Bagaria and Steve Todorcevic, editors, Set Theory, Centre de Recerca Matemàtica Barcelona, 2003-2004, pp. 209-230. Birkhäuser.

6. K. Liu. Stationary subsets of $\left[\aleph_{\omega}\right]^{<\aleph_{n+1}}$. J. Symbolic Logic, 58(4):1201-1218, 1993. MR 1253918 (94k:03059)

7. K. Liu and S. Shelah. Cofinalities of elementary substructures of structures on $\aleph_{\omega}$. Israel J. Math., 99:189-205, 1997. MR 1469093 (98m:03100)

Mathematisches Institut, Universität Bonn, Beringstrasse 1, D 53115 Bonn, Germany

E-mail address: koepke@math.uni-bonn.de 